\title{
MINER: exploratory analysis of gene interaction networks by machine learning from expression data Sidath Randeni Kadupitige ${ }^{\dagger 1,2,3}$, Kin Chun Leung ${ }^{\dagger 1,2}$, Julia Sellmeier ${ }^{\dagger 1}$, Jane Sivieng ${ }^{1}$, Daniel R Catchpoole $e^{3,4}$, Michael E Bain*1 and Bruno A Gaëta*1,2
}

\author{
Addresses: ${ }^{1}$ School of Computer Science and Engineering, The University of New South Wales, Sydney, NSW, 2052, Australia, \\ ${ }^{2}$ School of Biotechnology and Biomolecular Sciences, The University of New South Wales, Sydney, NSW, 2052, Australia, ${ }^{3}$ The Tumour Bank, \\ The Oncology Research Unit, The Children's Hospital at Westmead, Westmead, NSW, 2145, Australia and ${ }^{4}$ The Oncology Department, The \\ Children's Hospital at Westmead, Westmead, NSW, 2145, Australia \\ E-mail: Sidath Randeni Kadupitige - h.randeni@gmail.com; Kin Chun Leung - kcle343@cse.unsw.edu.au; \\ Julia Sellmeier - julia.sellmeier@gmail.com; Jane Sivieng - jsivieng@cse.unsw.edu.au; Daniel R Catchpoole - DanielC@chw.edu.au; \\ Michael E Bain* - mike@cse.unsw.edu.au; Bruno A Gaëta* - bgaeta@unsw.edu.au \\ ${ }^{*}$ Corresponding author †Equal contributors
}

from Asia Pacific Bioinformatics Network (APBioNet) Eighth International Conference on Bioinformatics (InCoB2009) Singapore 7-II September 2009

Published: 3 December 2009

BMC Genomics 2009, I0(Suppl 3):SI7 doi: 10.1186/147I-2164-10-S3-S17

This article is available from: http://www.biomedcentral.com/I47I-2/64/I0/S3/SI7

(C) 2009 Kadupitige et al; licensee BioMed Central Ltd.

This is an open access article distributed under the terms of the Creative Commons Attribution License (http://creativecommons.org/licenses/by/2.0), which permits unrestricted use, distribution, and reproduction in any medium, provided the original work is properly cited.

\begin{abstract}
Background: The reconstruction of gene regulatory networks from high-throughput "omics" data has become a major goal in the modelling of living systems. Numerous approaches have been proposed, most of which attempt only "one-shot" reconstruction of the whole network with no intervention from the user, or offer only simple correlation analysis to infer gene dependencies.

Results: We have developed MINER (Microarray Interactive Network Exploration and Representation), an application that combines multivariate non-linear tree learning of individual gene regulatory dependencies, visualisation of these dependencies as both trees and networks, and representation of known biological relationships based on common Gene Ontology annotations. MINER allows biologists to explore the dependencies influencing the expression of individual genes in a gene expression data set in the form of decision, model or regression trees, using their domain knowledge to guide the exploration and formulate hypotheses. Multiple trees can then be summarised in the form of a gene network diagram. MINER is being adopted by several of our collaborators and has already led to the discovery of a new significant regulatory relationship with subsequent experimental validation.

Conclusion: Unlike most gene regulatory network inference methods, MINER allows the user to start from genes of interest and build the network gene-by-gene, incorporating domain expertise in the process. This approach has been used successfully with RNA microarray data but is applicable to other quantitative data produced by high-throughput technologies such as proteomics and "next generation" DNA sequencing.
\end{abstract}




\section{Background}

The development of high-throughput technologies for measuring RNA levels and estimating gene expression for large sets of genes has provided a new window into transcriptional regulation. RNA species that vary together under a range of conditions are likely to be under common regulation, and indeed, sets of "co-expressed" genes generated by clustering of microarray expression values have proven useful for identifying potential regulatory elements and transcription factor binding sites [1-5].

This type of analysis has been extended to look for patterns of expression correlation between genes resulting from regulatory relationships, for example increased RNA levels for a transcription factor leading to an increase in the RNA levels of the genes whose transcription is activated by this factor. Several approaches have been proposed to identify potential regulatory relationships, including [6-9]. These regulatory relationships can be visualised as a gene regulatory network graph [10], and this graph, in turn, can be further analysed in terms of global properties [11] and to identify network motifs such as feedforward loops, feedback loops etc [12].

A large number of algorithms based on machine learning and reverse engineering principles have been proposed to infer gene regulatory interactions from microarray data (reviewed in [13-15]). However none of these methods has been very successful, in part due to the large amount of experimental noise in microarray data, which can be particularly problematic for "black box" batch learning methods that infer the most likely gene regulatory network from microarray data with little or no consideration for additional biological information, and keep the human biologist out of the loop. Methods that integrate multiple sources of information (expression levels, biological annotation, protein levels etc) [16-18] are promising but face difficulty in capturing and integrating all the relevant biological information, and their complexity can be prohibitive for the biologist user.

We are proposing an alternative approach based on the philosophy of putting users in control of the process of exploring possible regulatory relationships in an interactive fashion and being able to integrate their biological knowledge with machine learning-based predictions of potential regulatory relationships. The standard paradigm is to visualize the very large networks implicit in high-throughput interaction data, then study sub-network interactions in detail. We invert this, going from individual interactions with target genes to construct a larger network centred on those genes, in an interactive process under biologist control. This approach is used in MINER (Microarray Interactive Network Exploration and
Representation), a web browser-based framework that integrates machine learning of potential regulatory relationships from microarray data, presentation of biological relationships based on Gene Ontology (GO) annotations [19], and integration of multiple analyses into a gene regulatory network model that can be the basis for new hypotheses and experiments. This combination of dependency learning, GO annotation distance and interactive visualisation provides a novel approach for investigating potential regulatory relationships in expression data which can complement standard approaches. MINER has been used by our collaborators to explore different data sets, leading to the identification of potential relationships that were subsequently validated experimentally.

\section{Results}

Interactive exploration of potential regulatory

\section{relationships}

MINER is a web-based framework that analyses microarray data to suggest likely hypotheses regarding regulatory relationships between genes surveyed in the dataset. The system-level data flow of MINER is shown in Figure 1 . The system is fully user-driven and provides a convenient interface and visualisation paradigm that allows the biologist to explore the dependencies and relationships of genes of interest. A typical user workflow is shown in Figure 2. The user uploads normalised microarray data in tabular or colon-delimited format, and then selects one or more target genes of interest in

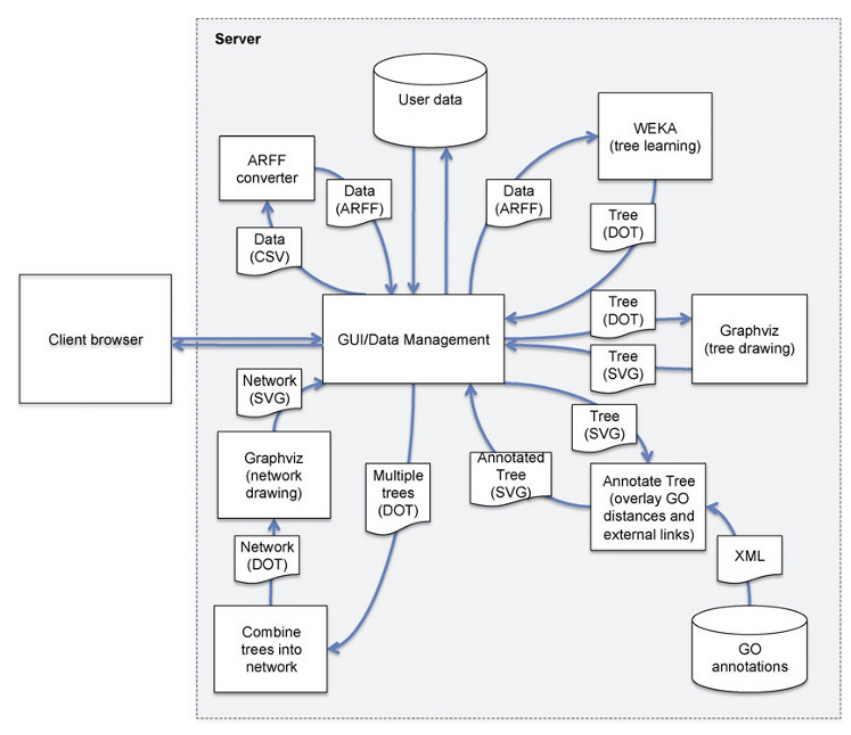

\section{Figure I}

MINER system-level data flow. Overview of data exchange between the various components of MINER. All the data passed to the Data management process are stored in the User data database. 


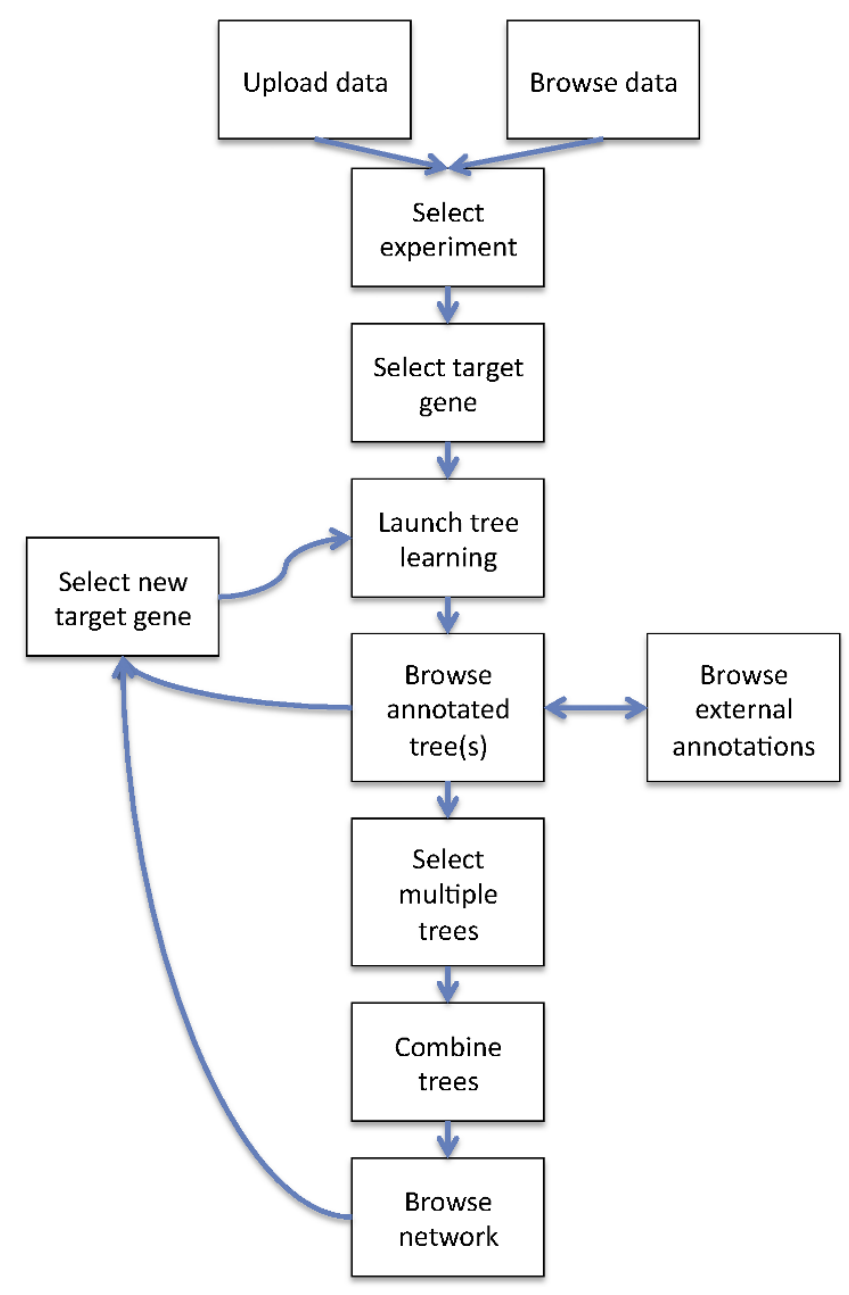

\section{Figure 2}

Typical user workflow in MINER. Flowchart

demonstrating the actions taken by the user in a typical MINER analysis. The user interface guides the user through the various steps. The user introduces biological expertise in the network inference process by choosing genes and trees to further analyse.

the dataset to launch the analysis. MINER applies decision tree, model tree or regression tree learning [20] to identify genes in the dataset whose expression can predict the expression of the target gene, and displays the result in the form of a decision tree for the user to explore. The user can mouse-over the nodes of the trees to display potential relationships based on common Gene Ontology [19] annotations between the node and other nodes in the tree, in the form of an overlaid graph (Figure 3). This visualisation is based on the ArcTree paradigm [21]. "Distances" between genes are calculated based on the graph distance between their GO annotation terms calculated using the CzekanowskiDice formula [22]. The distance between two genes is

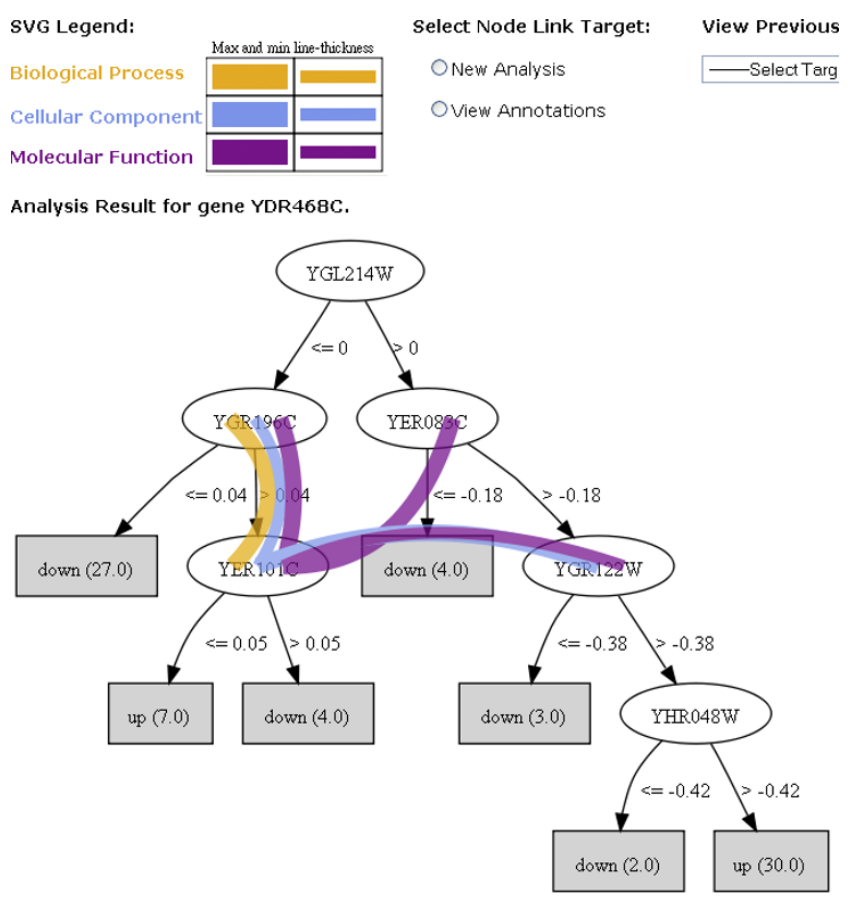

Figure 3

Example annotated tree (Arctree) produced by

MINER. Screenshot from the MINER GUI showing a decision tree of genes whose expression in the input microarray data set can predict the expression of the target gene YDR468C. For example, in this case, the tree indicates that YDR468C was down-regulated in the data set when the expression of YGL2 I4W had a normalised expression level of 0 or less and YGR I 96C had a normalised expression level of 0.04 or less. Placing the mouse over a node of the tree displays coloured arcs representing the "annotation distance" between the gene represented by this node and other genes in the tree. Genes who share more GO annotations are linked by thicker arcs. The colour of the arc corresponds to the GO category represented (Biological Process, Molecular Function, Cellular Component). For example, in this case, YGR I96C and YER IOIC share similar GO function, process and localisation annotations. Depending on the radio button setting, clicking on a node either displays external annotations on the corresponding gene or launches a new tree learning analysis using the corresponding gene as the new target.

represented by the thickness of the line connecting the two corresponding nodes in the tree, and the colour of the line represents the GO category (Molecular Function, Biological Process, Cellular Compartment) represented. Clicking on a node allows the user to display linked annotations in the Kyoto Encyclopaedia of Genes and Genomes (KEGG) database [23] or to launch a new analysis using the selected node as target gene, to build a new tree for this gene. 
After decision trees have been built for multiple genes, MINER allows summarising the multiple trees into a network graph, by representing each potential regulatory relationship seen in a decision tree as an edge in a graph (Figure 4), based on the algorithm given in Figure 5. These decision trees, networks and overlaid annotations can assist the user in the formulation of new hypotheses regarding the regulation of the target gene, which can be subsequently tested experimentally.

The tree-learning approach was inspired by the work of Brazma and others [24] and was extended by us to work on real-valued data using regression and model trees [25]

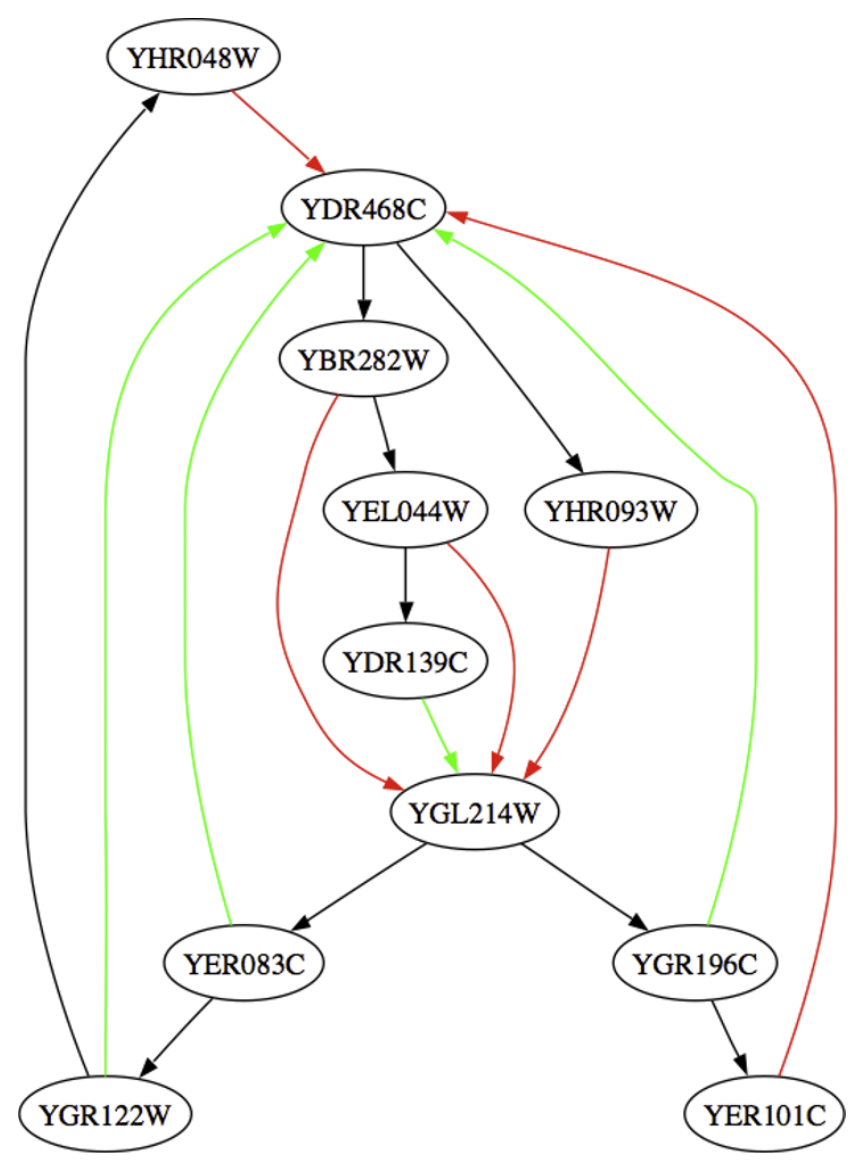

\section{Figure 4}

Example MINER gene interaction network. Screen capture from MINER showing a section of a gene interaction network generated by MINER from two trees, one of which appears in Figure 3. Regulatory relationships identified in the learned tree are represented as edges in the network, with interactions shown in either red (for up-regulation) or green (for down-regulation) acting on a target gene; edges shown in black are between two non-target genes and indicate that there may be a regulatory relationship or interaction between these genes but the nature of this relationship is not inferable from the component trees.

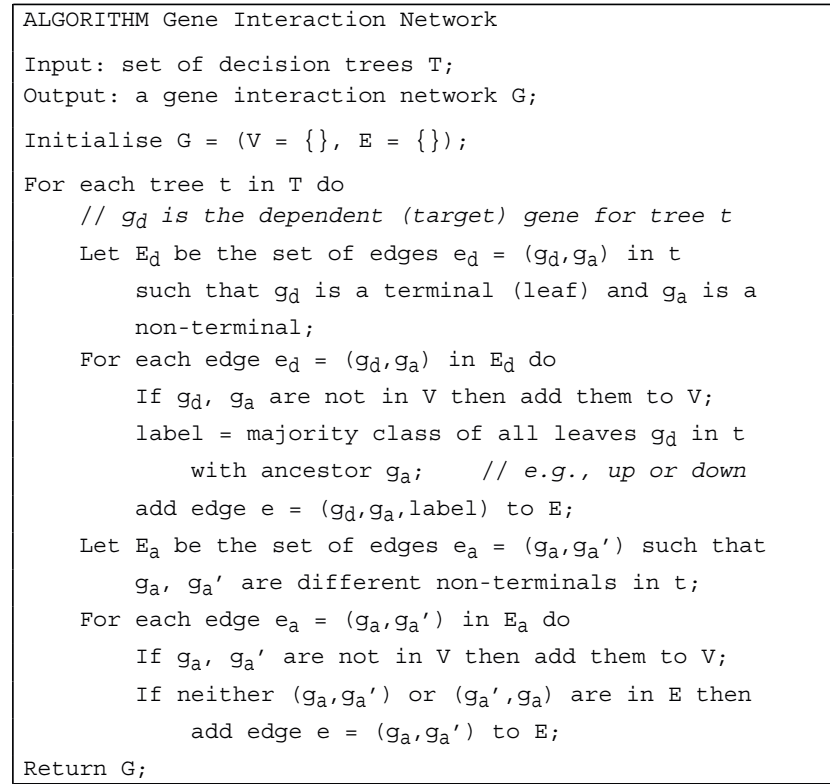

\section{Figure 5}

Algorithm: Gene Interaction Network from Decision Trees. MINER uses this algorithm to construct a Gene Interaction Network from a set of Decision Trees. Both input trees and the output network are vertex- and edgelabelled directed graphs where vertices represent conditions on genes. However, in each type of graph the vertex and edge label sets differ.

where it was applied to yeast microarray data. Further extensions, particularly the use of a relational database, graphical user interface, support for gene interaction network construction and Gene Ontology distance functions were implemented in a number of follow-up projects.

\section{Evaluation}

Due to the large data requirements the MINER system is currently not publicly available on the web but it has been implemented for two of our collaborators, in one case for $S$. cerevisiae data, and in the other for an acute lymphoblastic leukaemia microarray dataset [26]. In the latter case, MINER suggested a new significant regulatory relationship in leukemic cells that was subsequently validated experimentally (Guo D, O' Sullivan M, Henry M, Fong A, Kiiveri H, Stone G, Randeni H, Gaeta B, Bain $\mathrm{M}$ and Catchpoole D - manuscript in preparation).

MINER relies on human intervention to guide the network-building process and as such cannot be evaluated in comparison to fully automated "one-shot" network inference algorithms. However, as part of previous work [25] we evaluated the tree learning methods used in MINER on the standard yeast cell 
cycle microarray data set [27]. In our study three methods of tree learning were used: decision tree learning, where the dependent variable is discrete-values, and two methods of numeric prediction, regression and model trees. All systems were implemented in the WEKA toolkit $[20]$ and learning performance was estimated using 10 -fold cross-validation. Tree learning was performed for each of the twenty target genes identified by Soinov et al. [24].

For decision tree learning we found a mean accuracy of $72 \%$, with twelve out of twenty trees scoring above $70 \%$ accuracy, and all scoring above 50\%. Correlations were above 0.7 for five (resp. eight) out of twenty for regression (resp. model) trees, with mean correlations of 0.5 (resp. 0.6) over all twenty target genes. Given that the data is noisy with a low number of samples, high number of genes, and many missing values, these results are as expected.

Subsequent experiments (unpublished data) using a network simulator to generate synthetic microarray data with artificial noise has shown that the tree learning in MINER can recover the gene dependencies embedded in simple network motifs such as feed-forward loops. In [25] we also found network links between genes across different trees, as would be discovered in automatic construction of gene interaction networks.

\section{Discussion}

\section{Representing interaction networks}

As is common in current genome-scale informatics, the fundamental object for MINER is the "gene", although this can actually refer variously to gene products such as transcripts, proteins, intergenic (promoter) regions, etc. A network may be formalised as a graph $G=(V, E)$, where each vertex in the set $V$ denotes a gene, and each edge in the set $E$ represents some kind of interaction between genes. Edges may be directed or undirected, and may have labels, e.g., to distinguish between different types of interactions.

\section{Using a machine learning toolkit}

MINER uses the WEKA machine learning toolkit [20] for tree learning. The advantage of a general-purpose machine learning toolkit in the exploratory analysis of genome-scale interaction data is the ease and rapidity with which many different forms of data mining can be performed. For example, it is possible to move quickly from simple visualizations of the data and summary statistics to sophisticated methods such as non-linear multi-variate regression or high-dimensionality kernelbased classifier learning.
Since the predominant mode of analysis in MINER is exploratory rather than hypothesis testing, it is necessary to have powerful methods capable of detecting the faint signals present in noisy data such as microarrays. Although these may increase the risk of Type 1 errors (i.e., false positives, suggesting interactions which in fact have no biological basis), it is understood that any detected interaction will be subject to further analysis by different techniques before they can be accepted. There is also a role in this process for integrating potential interactions with other sources of data to increase confidence. On the positive side there are many advantages in reverse engineering networks by interactively tracing out patterns of influence of genes on other genes using the powerful means of signal detection implemented in machine learning methods.

Non-linear regression of multiple genes on a target using model tree learning subsumes techniques such as correlation-based construction of co-expression matrices. This is important since regulatory relationships may be non-linear. In particular, this representation can learn context-dependent (potentially regulatory) relationships: as an example, we could have that given gene $A>1.3$ and gene $B<-0.9$ then the dependence of genes $C, D$, and $E$ on target $F$ is given by the linear regression equation $F=-0.2 C+2.3 D+0.1 E+0.7$. Such context sensitivity has the potential to detect regulatory signals in data that could be missed by simply finding the pairwise correlations of genes $A, . ., E$ with target gene $F$.

Tree learning methods also perform attribute (variable) selection during the learning process, finding a subset of genes implicated in potential regulatory relationships with a target, enabling inspection by a biologist, since typically this represents only a small subset of the whole genome. The potential for overfitting can be controlled by user-driven pruning built into the algorithms. Other learning methods such as high-dimensionality kernel methods can be applied to the same data sets; in this case feature selection can be applied by either pre-processing the data, or post-processing the learned model [28].

\section{Network construction from multiple trees}

Transforming a set of trees (e.g., see Figure 3), each of which encodes a disjunction of conjunctive rules on the conditions (gene expression levels) under which a single target gene is expressed, to a network that captures the combination of regulatory dependencies between multiple genes in a user-friendly way is not straightforward. We adopted a level-wise approach (Figure 5). At the first level all the trees learned from the expression data are retained, since they capture the details of the regulatory relationships of genes on their targets. 
A higher-level network is then constructed by combining the trees at the first level and removing some of the detail. Recall that both levels are expanded only as the user explores the space of target genes.

At the network level, the goal is not to provide the detailed logic of combinations of condition-specific gene regulation, but rather to show the general organisation of regulatory gene interactions. To do this we use the structure of the trees. Parents of terminal (leaf) nodes are more closely linked with their target genes and edges are labelled to denote the principal regulatory effect (e.g., up or down). Edges linking non-terminal (internal) nodes are then added without labels to denote an indirect regulatory interaction. Note that functionally these relationships may be just as important. However, this structures the network and reduces clutter in the visualization. Since all details are retained in the trees at the lower level, no information is lost. An example of such a network is shown in Figure 4.

\section{Integration of heterogeneous data sources}

Gene Ontology: MINER uses a distance measure on the GO annotation of pairs of genes [22] to evaluate their biological relatedness. This is currently implemented at the level of individual trees, but could be easily incorporated into network edges as well.

Kyoto Encyclopedia of Genes and Genomes (KEGG): each gene appearing in an internal node of a decision tree is annotated with a species-specific URL denoting its entry in the KEGG GENES database. This is then included in the SVG file that displays the decision tree graphically in the browser interface. When the user clicks on a node in the tree, the browser executes a query to open the gene's annotation page and display details of its name, sequence, and other annotation using KEGG's DBGET method.

Other sources of expression data: Since tree induction methods are non-parametric they may be applied to other data sources, as long as they are in a similar format to mRNA expression data, such as data from nextgeneration sequencing data, proteomics or glycomics. This is because data generated in the form of (absolute or relative) abundances, such as from high-throughput mass spectrometry are similar to microarray data in the sense of being an indirect measure of concentration of gene products or other molecules. However, this is left for future work since so far we have only applied MINER to microarray data.

\section{Related work}

A large number of methods have been proposed to infer whole gene regulatory networks from gene expression data (reviewed in [13-15]). These methods all apply a "one-shot" paradigm that can lack transparency for the end user and does not allow the use of the biologist's domain knowledge. MINER differs from most approaches through its interactivity that allows the user to explore the data and generate testable hypotheses in the process.

Other interactive methods fall into two categories: network visualisation tools that can incorporate some network inference algorithm, and interactive data mining applications.

In the first category, SEBINI [29] is designed to be a framework to support testing of network inference algorithms using synthetic and other data sets. However, it has a limited number of inference methods incorporated, and cannot support the two level approach we have adopted. It also does not seem to be actively under development. ToPNet [30] adopts the Petri Net formalism to represent interactions, which is more flexible than simple graphs, particularly for metabolic reactions. However, it does not support any data mining methods for network inference, and it is no longer supported. Cytoscape [31] is a widely used visualisation and integration package that supports some network inference plug-ins (for example [32,33]). All of these plug-ins perform a global network inference based on uni-variate correlation rather than the gene-by-gene approach of MINER that uses more involved multi-variate non-linear tree learning.

In the second category, SysNet [34] combines visualization and exploratory data analysis, however its network inference is restricted to standard methods of correlation. Unlike MINER, SysNet infers a global network first then allows the user to drill down to inspect properties of individual nodes rather than building the network from individual relationships.

\section{Conclusion}

MINER combines advanced machine learning techniques with a "bottom-up" interactive approach to inferring gene interaction networks from gene expression data. This approach differs from most methods that attempt to reconstruct the whole network in one operation and are not very transparent to the end user, and from interactive methods that are based on relatively simple expression correlation and clustering. The MINER approach allows biologists to examine the program's hypotheses as they are generated and incorporate their own biological knowledge into the interaction network exploration process. The tree learning paradigm provides explicit descriptions of regulatory dependencies with supporting 
evidence for the user to examine. This interactive exploration approach has already resulted in the discovery of new regulatory relationships that were subsequently validated experimentally. MINER has been used with gene expression data obtained from microarray experiments but can be applied to any highthroughput molecular abundance data including those resulting from new sequencing technologies and from proteomics analyses.

\section{Methods}

\section{Component packages}

MINER is implemented using PHP [35] with some components in Perl [36] It uses the MySQL RDBMS [37] for storing user data, results and GO annotations and relationships. The decision tree learning component of MINER uses the J48 algorithm implemented in WEKA (version 3.4.8) [20] with default parameters $(C=0.25$, $M=2$ ). Regression and model tree learning uses WEKA's M5Prime implementation with default parameter settings. Tree and network diagrams are produced using the Graphviz package [38].

\section{Data formats}

Microarray data can be uploaded to MINER in tabular or comma-delimited format, and are converted into ARFF (Attribute-Relation File Format) for input into WEKA. Trees are produced by WEKA in DOT format and converted by Graphviz into images in SVG (Scalable Vector Graphics) format [39] for interactive visualisation. Since MINER's graphical outputs (trees and networks) are in the SVG format, a suitable browser rendering component is required for visualization. Current versions of all major web browsers except Microsoft's Internet Explorer have built-in support for rendering SVG graphics. Users of Internet Explorer can download a plugin to enable SVG support.

\section{User interface design methodology}

The MINER graphical user interface was developed using standard UI development methodology. A range of visualisation paradigms were proposed and non-functional mock-ups were developed. The mockups were presented to a focus group of potential end users whose feedback guided the selection and refinement of the final visualisation paradigm. The design process applied human-computer interaction and ergonomics principles. For example, colours were selected to be easily distinguished even by most colour-blind users.

\section{List of abbreviations used}

GO: Gene Ontology; KEGG: Kyoto Encyclopaedia of Genes and Genomes; ARFF: Attribute-Relation File Format; SVG: Scalable Vector Graphics.

\section{Competing interests}

The authors declare that they have no competing interests.

\section{Note}

Other papers from the meeting have been published as part of BMC Bioinformatics Volume 10 Supplement 15, 2009: Eighth International Conference on Bioinformatics (InCoB2009): Bioinformatics, available online at http://www.biomedcentral.com/1471-2105/10? issue $=\mathrm{S} 15$.

\section{Authors' contributions}

SRK implemented the basic functionality of the program including decision trees and network visualization. KCL implemented annotated tree visualization, model trees and regression trees. JSe gathered software requirements and designed and tested the visualization paradigm. JSi contributed to the programming and implementation of the system. DRC provided data sets for testing and user feedback. MEB and BAG collaborated on the conception of the project, led the group and drafted the manuscript, with MEB focusing on machine learning analysis and BAG on user interface and implementation. All authors read and approved the final manuscript.

\section{Acknowledgements}

Part of the work on MINER by SRK and DRC was funded by The Australian Rotary Health Research Fund, the Oncology Children's Foundation and Kayaking for Kemo Kids.

This article has been published as part of BMC Genomics Volume 10 Supplement 3, 2009: Eighth International Conference on Bioinformatics (InCoB2009): Computational Biology. The full contents of the supplement are available online at http://www.biomedcentral.com//47/-2/64/I0? issue $=\mathrm{S} 3$.

\section{References}

I. Tompa M, Li N, Bailey TL, Church GM, De Moor B, Eskin E, Favorov AV, Frith MC, Fu $Y$ and Kent WJ, et al: Assessing computational tools for the discovery of transcription factor binding sites. Nat Biotechnol 2005, 23:137-| 44

2. Defrance $M$ and Touzet $H$ : Predicting transcription factor binding sites using local over-representation and comparative genomics. BMC Bioinformatics 2006, 7:396-406.

3. Thijs G, Marchal K, Lescot M, Rombauts S, De Moor B, Rouze P and Moreau $Y$ : A Gibbs sampling method to detect overrepresented motifs in the upstream regions of coexpressed genes. J Comput Biol 2002, 9:447-464.

4. Aerts S, Thijs G, Coessens B, Staes M, Moreau $Y$ and Moor BD: Toucan: deciphering the cis-regulatory logic of coregulated genes. Nucleic Acids Res 2003, 31:1753-1764.

5. Van Helden J: Regulatory Sequence Analysis Tools. Nucleic Acids Res 2003, 31:3593-3596.

6. Stuart JM, Segal E, Koller D and Kim SK: A gene-coexpression network for global discovery of conserved genetic modules. Science 2003, 302:249-255.

7. Bar-Joseph Z, Gerber G, Lee T, Rinaldi N, Yoo J, Robert F, Gordon D, Fraenkel E, Jaakkola T and Young R, et al: Computational discovery of gene modules and regulatory networks. Nat Biotechnol 2003, 21:1337-1342.

8. Haverty $P$, Frith $M$ and Weng Z. CARRIE web service: automated transcriptional regulatory network inference and interactive analysis. Nucleic Acids Res 2004, 32:W2 13-W216. 
9. Friedman N: Inferring Cellular Networks Using Probabilistic Graphical Models. Science 2004, 303:799.

10. Hu Z, Mellor J, Wu J, Kanehisa M, Stuart JM and DeLisi C: Towards zoomable multidimensional maps of the cell. Nat Biotechnol 2007, 25:547-554.

II. Carlson M, Zhang B, Fang Z, Mischel P, Horvath S and Nelson S: Gene connectivity, function, and sequence conservation: predictions from modular yeast co-expression networks. BMC Genomics 2006, 7:40-54.

12. Lee TI, Rinaldi NJ, Robert F, Odom DT, Bar-Joseph Z, Gerber GK, Hannett NM, Harbison CT, Thompson CM and Simon I, et al: Transcriptional regulatory networks in Saccharomyces cerevisiae. Science 2002, 298:799-804.

13. Markowetz F and Spang R: Inferring cellular networks-a review. BMC Bioinformatics 2007, 8(Suppl 6):S5.

14. Schlitt $T$ and Brazma $A$ : Current approaches to gene regulatory network modelling. BMC Bioinformatics 2007, 8(Suppl 6):S9.

15. Li H, Xuan J, Wang $Y$ and Zhan $\mathrm{M}$ : Inferring regulatory networks. Front Biosci 2008, 13:263-275.

16. Troyanskaya OG, Dolinski K, Owen AB, Altman RB and Botstein D: A Bayesian framework for combining heterogeneous data sources for gene function prediction (in Saccharomyces cerevisiae). Proc Natl Acad Sci USA 2003, 100:8348-8353.

17. Beyer A, Workman C, Hollunder J, Radke D, Möller U, Wilhelm T and Ideker $\mathrm{T}$ : Integrated assessment and prediction of transcription factor binding. PLoS Comput Biol 2006, 2:e70.

18. Zhu J, Zhang B, Smith EN, Drees B, Brem RB, Kruglyak L Bumgarner RE and Schadt EE: Integrating large-scale functional genomic data to dissect the complexity of yeast regulatory networks. Nat Genet 2008, 40:854.

19. Ashburner M, Ball CA, Blake JA, Botstein D, Butler H, Cherry JM, Davis AP, Dolinski K, Dwight SS and Eppig JT, et al: tool for the unification of biology. The Gene Ontology Consortium. Nat Genet 2000, 25:25-29.

20. Witten IH and Frank E: Data mining practical machine learning tools and techniques. Morgan Kaufmann series in data management systems Amsterdam; Boston, MA: Morgan Kaufman; 22005.

21. Neumann P, Schlechtweg S and Carpendale S: Arctrees: Visualizing relations in hierarchical data. Proc of Eurographics 2005 - IEEE VGTC Symp on Visualization 2005, 53-60.

22. Brun C, Chevenet F, Martin D, Wojcik J, Guenoche A and lacq B: Functional classification of proteins for the prediction of cellular function from a protein-protein interaction network. Genome Biol 2003, 5:R6.

23. Kanehisa M, Goto $S$, Kawashima $S$ and Nakaya A: The KEGG databases at GenomeNet. Nucleic Acids Res 2002, 30:42-46.

24. Soinov LA, Krestyaninova MA and Brazma A: Towards reconstruction of gene networks from expression data by supervised learning. Genome Biol 2003, 4:R6.

25. Bain M and Gaëta B: Learning Quantitative Gene Interactions from Microarray Data. ADM 2003: Proc of the 2nd Australian Workshop on Data Mining University of Technology, Sydney: Simoff S Williams G, Hegland M 2003, 35-49.

26. Catchpoole D, Guo D, liang $\mathrm{H}$ and Biesheuvel C: Predicting outcome in childhood acute lymphoblastic leukemia using gene expression profiling: Prognostication or protocol selection? Blood 2008, I II:2486-2487.

27. Spellman PT, Sherlock G, Zhang MQ, lyer VR, Anders K, Eisen MB, Brown PO, Botstein D and Futcher B: Comprehensive identification of cell cycle-regulated genes of the yeast Saccharomyces cerevisiae by microarray hybridization. Mol Biol Cell 1998, 9:3273-3297.

28. Guyon I and Elisseeff A: An introduction to variable and feature selection. The J Mach Learn Res 2003, 3: I I57-I I82.

29. Taylor RC, Shah A, Treatman C and Blevins M: SEBINI: Software Environment for Blological Network Inference. Bioinformatics 2006, 22:2706-2708.

30. Hanisch D, Sohler F and Zimmer R: ToPNet-an application for interactive analysis of expression data and biological networks. Bioinformatics 2004, 20: |470-|47|

31. Cline MS, Smoot M, Cerami E, Kuchinsky A, Landys N, Workman C Christmas R, Avila-Campilo I, Creech M and Gross B, et al: Integration of biological networks and gene expression data using Cytoscape. Nat Protoc 2007, 2:2366-2382.

32. Morcos F, Lamanna C, Sikora M and Izaguirre J: Cytoprophet: a Cytoscape plug-in for protein and domain interaction networks inference. Bioinformatics 2008, 24:2265-2266.

33. Margolin A, Nemenman I, Basso K, Wiggins C, Stolovitzky G, Dalla Favera R and Califano A: ARACNE: an algorithm for the reconstruction of gene regulatory networks in a mammalian cellular context. BMC Bioinformatics 2006, 7(Suppl I):S7.

34. Zhang M, Ouyang Q, Stephenson A, Kane M, Salt D, Prabhakar S, Burgner J, Buck C and Zhang X: Interactive analysis of systems biology molecular expression data. BMC Systems Biology 2008, 2:23.

35. PHP: Hypertext Preprocessor. http://www.php.net

36. The Perl Directory. http://www.perl.org.

37. MySQL:: The world's most popular open source database. http://www.mysql.com.

38. Graphviz. http://www.graphviz.org.

39. Scalable Vector Graphics (SVG). http://www.w3.org/Graphics/ $\underline{\text { SVG/. }}$
Publish with BioMed Central and every scientist can read your work free of charge

"BioMed Central will be the most significant development for disseminating the results of biomedical research in our lifetime. "

Sir Paul Nurse, Cancer Research UK

Your research papers will be:

- available free of charge to the entire biomedical community

- peer reviewed and published immediately upon acceptance

- cited in PubMed and archived on PubMed Central

- yours - you keep the copyright
BioMedcentral 